\title{
Influence of Diet and Dietary Habits in Caries \\ Occurrence Among 12- to 15-year-old Adolescents in Shanghai
}

\section{Hua-xing XU}

Department of Endodontics, Shanghai Stomatological Hospital \& Shanghai Key Laboratory of Craniomaxillofacial Development and Diseases, Fudan University

\section{Chun-yan LUO}

Shanghai Municipal Center For Disease Control Prevention

\section{Xiao-gang FENG}

Shanghai Municipal Center For Disease Control Prevention

\section{Hao ZHANG}

Department of Preventive dentistry, Shanghai Stomatological Hospital \& Shanghai Key Laboratory of Craniomaxillofacial Development and Diseases, Fudan University

\section{Yi-wei Jiang}

Department of Preventive dentistry, Shanghai Stomatological Hospital \& Shanghai Key Laboratory of Craniomaxillofacial Development and Diseases, Fudan University

Jin YU

Department of Preventive dentistry, Shanghai Stomatological Hospital \& Shanghai Key Laboratory of Craniomaxillofacial Development and Diseases, Fudan University

\section{Hu-ning Wang}

Department of Preventive dentistry, Shanghai Stomatological Hospital \& Shanghai Key Laboratory of Craniomaxillofacial Development and Diseases, Fudan University

\section{Dong-xin DA}

Department of Preventive dentistry, Shanghai Stomatological Hospital \& Shanghai Key Laboratory of Craniomaxillofacial Development and Diseases, Fudan University

\section{Dong-ling YANG}

Shanghai Municipal Center For Disease Control Prevention

\section{Li-jin SUN}

Shanghai Municipal Center For Disease Control Prevention

\section{Ying ZHANG}

Department of Preventive dentistry, Shanghai Stomatological Hospital \& Shanghai Key Laboratory of Craniomaxillofacial Development and Diseases, Fudan University

Xiao-li ZENG ( $\square$ lylyz97@163.com) 
Department of Preventive dentistry, Shanghai Stomatological Hospital \& Shanghai Key Laboratory of Craniomaxillofacial Development and Diseases, Fudan University

\section{Research Article}

Keywords: diet and dietary habits, decayed, missing, and filled teeth (DMFT), Poor eating habits

Posted Date: February 1st, 2021

DOl: https://doi.org/10.21203/rs.3.rs-154187/v1

License: (9) This work is licensed under a Creative Commons Attribution 4.0 International License. Read Full License 


\section{Abstract}

Background: The purpose of this study was to investigate the influence of diet and dietary habits on dental caries status in the permanent teeth of 12-to 15-year-old adolescents in Shanghai, China.

Methods: We conducted a cross-sectional investigation among 11,599 school students aged 12- to 15 years from Shanghai, China. We distributed self-administered questionnaires and performed clinical dental examinations according to WHO guidelines to obtain information about tooth-brushing habits, diet, dietary habits, and dental caries status. We generated a logistic regression model including dental cariesrelated variables to ascertain the potential risk factors associated with dental caries.

Results: The caries prevalence and number of decayed, missing, and filled teeth (DMFT) among the 11,59912 - to 15 -year-old students surveyed were $26.2 \%$ and $0.59 \pm 1.314$, respectively. The caries prevalence and DMFT of girls were significantly higher than those of boys $(P<0.05)$. Caries prevalence and DMFT also increased with age $(P<0.05)$. There was no significant difference $(P>0.05)$ in caries prevalence and mean DMFT among groups with different tooth-brushing habits. Logistic regression analysis suggested that the possible risk factors for dental caries included female sex, older age, consuming sugary drinks at least once daily, consuming vegetables once daily or less, and skipping breakfast, with ORs ranging from 1.124 to $1.88(P<0.05)$.

Conclusion: The caries prevalence among 12- to 15-year-old school students in Shanghai was lower than that reported nationally and in some other developing or developed countries. Diet and dietary habits were closely related to dental caries among adolescents. Poor eating habits (higher sugary drink intake, lower vegetable intake, and skipping breakfast) in the developmental age may be a significant caries coefficient in adolescents. A longitudinal study with full consideration of baseline variables is warranted in the Chinese population.

\section{Introduction}

Dental caries is one of the most prevalent chronic diseases and an alarming public health problem worldwide [1]. It is a multifactorial disease characterized by the destruction of dental tissues due to demineralization from the acids generated by bacterial plaque. The "classic" relationship between diet and dental caries has been reviewed in recent years, and the relative importance of sugar consumption in caries etiology is now seen as a modifying risk factor rather than primary causative factor [2-6]. This relationship has also been confirmed by recent reports from cross-sectional and longitudinal studies in different countries [7-12]. Furthermore, studies have suggested that individual eating behavior plays a larger role in caries development than diet itself $[13,14]$. Unhealthy lifestyle factors such as skipping meals and choosing nutrient-poor foods are common among the vulnerable adolescent group $[15,16]$. Children and adolescents generally prefer sweetened foods and soft drinks rich in carbohydrates, and thus are at higher risk for caries development [17]. With the known cultural difference, where the Chinese diet is different from a western diet, and with few studies addressing this issue there arises the need to 
explore this concept of the role of dietary habits and diet in caries occurrence in China. Therefore, it is important to obtain information on diet and eating habits in adolescents to target and improve the dental health of this population. Our aim was to investigate the role of dietary habits and diet in caries occurrence in a cross-sectional study of 12- to 15-year-old school students in Shanghai (December 2018May 2019) and to relate the findings to dental caries development.

\section{Study Population And Methodology 2.1 Study population:}

This cross-sectional study used data collected from the Monitoring and Intervention Project of Common Diseases of Students in Shanghai conducted in 2019. Based on our inclusion criteria, we recruited children aged 12-15 years, with a target population of junior high school students. Three junior high schools were selected randomly from each of the 17 districts of Shanghai. We monitored the entire class in units, and selected at least 80 students from each grade, supplementing the shortfall with students from nearby schools. A total of 11599 school students aged 12-15 years formed our study sample. This study was reviewed and approved by the Independent Ethics Committee of Shanghai Municipal Center for Disease Control and Prevention. Written informed consent was obtained from the parents or guardians of all participants in the study. The STROBE guidelines (Strengthening the Reporting of Observational Studies in Epidemiology) were followed [18].

\subsection{Diet, dietary habits and oral hygiene routines}

We collected data on diet, eating, and tooth-brushing habits with the aid of a self-reported questionnaire. The questionnaire asked about tooth-brushing frequency, skipping meals, and dietary habits, as possible risk factors for dental caries. The intakes of sugary drinks, confectioneries, fried foods, fresh fruits, and vegetables were queried in separate items, and scored by frequency as "never," "<1 time/day," "1 time/day," or ">2 times/day." Breakfast habits were scored according to frequency as "occasionally" or "every day." The questions regarding oral hygiene focused on tooth-brushing routines. Students completed the questionnaire in small groups in a quiet setting at school.

\subsection{Dental examinations}

Clinical dental examinations were performed in schools using a $0.5-\mathrm{mm}$ ball-ended community periodontal index probe and a disposable dental mirror. We used the World Health Organization (WHO) Oral Health Survey criteria to diagnose caries and made a positive diagnosis only when both visual and tactile criteria were met simultaneously. We did not perform a bitewing x-ray examination or use other radiographs in the caries assessment. We used WHO coding to characterize the caries, and calculated decayed-missing-filled teeth (DMFT) of from 28 teeth in the permanent dentition.

\subsection{Statistical analyses}


We analyzed the data using IBM SPSS (version 20.0, Armonk, NY, USA). The DMFT index is represented as mean \pm standard deviation. Chi square tests were used to compare the significant differences in permanent caries prevalence among children of different genders, ages, diets/dietary habits, and oral hygiene routines [2]. A Mann-Whitney U test was used to compare significant differences in the DMFT index among children of different genders, ages, dietary habits, and oral hygiene routines. To control for potential confounding effects, logistic regression was performed. The permanent caries variable was included as a dependent variable in the multivariable logistic regression models, and variables such as gender, age, diet, and dietary habits were included simultaneously as independent variables. The level of statistical significance was set at 0.05 for all two-sided statistical tests [19].

\section{Results}

\subsection{General characteristics of the study sample}

Table 1 lists the characteristics of the study population, including variables related to oral hygiene. The sample comprised 11,599 school students aged 12-15 years from the city of Shanghai, of which 5968 were male (51.5\%), and 5,631 were female (45.0\%). In addition, 2605 subjects were 12 years old (22.5\%), 2598 subjects were 13 years old (22.4\%), 2523 subjects were 14 years old (21.8\%), and 3873 subjects were 15 years old (33.4\%). A majority of the teenagers (75.2\%) brushed their teeth twice a day, $24.7 \%$ of teenagers brushed their teeth once a day, and the remaining teenagers (1\%) brushed their teeth occasionally or never.

\subsection{Diet and dietary habits}

In the week before the survey day, $77.5 \%$ of participants consumed sugary drinks less than once a day, $75.5 \%$ ate sweets less than once a day, $83.7 \%$ ate fried food less than once a day, $53.6 \%$ ate fresh fruit once a day, and $50.3 \%$ ate vegetables at least twice a day. In addition, $18.9 \%$ of participants reported irregular breakfasts, as shown in Table 2. The proportion of subjects who reported irregular breakfasts increased with age, reaching the highest at 14 years of age (21.8\%), and dropping slightly at 15 years of age (20.4\%), as shown in Figure 1.

\subsection{Caries Status}

The total caries prevalence and mean DMFT were $26.2 \%$ and $0.59 \pm 1.314$, respectively. The caries prevalence rate and mean DMFT of girls $(30.6 \%, 0.74 \pm 1.511)$ were significantly $(P<0.001)$ higher than that of boys $(22.1 \%, 0.45 \pm 1.087)$. In addition, the caries prevalence rate and mean DMFT of 15-year-olds $(32.0 \%, 0.78 \pm 1.540)$ was significantly $(P<0.001)$ higher than that of other age groups. There was no significant difference $(P>0.05)$ in caries prevalence and mean DMFT among groups with different brushing habits (Table 3 ).

\subsection{The association between diet/dietary habits and caries occurrence}


Table 4 shows the results of using a backward logistic regression model to analyze the association between dental caries prevalence and related risk factors. Five risk factors were present in the final results: female sex, older age, consuming sugary drinks at least once daily, eating vegetables once daily or less, and skipping breakfast. These were the most important risk factors for dental caries, with ORs ranging from 1.124 to $1.88(\mathrm{P}<0.05)$.

\section{Discussion}

Oral health-related quality of life is significant in adolescents, as many of their social and psychological coping skills are still developing [20]. The present study assessed the relationship between dietary habits and caries incidence in 12- to 15-year-old adolescents in Shanghai, China.

The caries prevalence and mean DMFT among 12-year-olds were $19.7 \%$ and 0.59 , respectively. These values are much lower than those seen nationally (41.9\% and 1.04, respectively) [21], and also lower than those seen in other regions like Zhejiang province, China $(40.9 \% ; 0.98)$ [22], Sichuan province, China $(37.2 \% ; 0.86)$ [23], Ambala, India (34.3\%; 0.82) [24], and Galicia, Spain $(39.6 \% ; 0.98)$ [25]. The caries prevalence showed an upward trend with increasing age, ranging from $19.7 \%$ among 12 -year-olds to $32 \%$ among 15-year-olds. This may be attributed to the continuous and cumulative process of caries development as well as the increase in erupting teeth with advancing age. Other studies have also reported a similar caries prevalence among these age groups $[25,26]$. In our study, the 14- and 15-year-old groups showed the highest overall caries prevalence, signifying a continued decline in oral health. The prevalence of dental caries and the mean of DMFT were higher in females than in males. This may be attributed to the earlier eruption of teeth in females, which could lead to prolonged exposure to cariogenic acids in the oral environment. However, other evidence suggests that the higher caries susceptibility among females may also be attributed to changes in salivary rates and composition induced by genderspecific hormonal fluctuations [27].

Tooth brushing is a relatively affordable method of reducing dental caries risk, mainly via exposure to fluoride from toothpaste and mechanical plaque removal $[14,28]$. The association between toothbrushing frequency and dental caries may differ depending on the country. Bernabé et al. [29] reported a reduced 4-year net increment in tooth decay via tooth-brushing twice a day or more compared with once a day or less in a Finnish population. Similarly, Rothen et al. [30] demonstrated that brushing teeth with a fluoride toothpaste twice daily reduced the incidence of caries in northwest American dental patients. However, in this study, the frequency of tooth-brushing was not associated with the incidence of dental caries. This finding supports a previous cross-sectional study in a Japanese population [31], possibly due to the design of the study questionnaire. In addition to the frequency of tooth-brushing, the quality of tooth-brushing is also important. Using the current questionnaire, it is impossible to know whether a subject has adopted the correct method of brushing and sufficient time for tooth-brushing. These factors may also affect the incidence of dental caries [32]. 
Moreover, beverage consumption patterns have changed in the past 10 years. The consumption of sugarsweetened beverages has increased annually, while the consumption of water and milk has gradually decreased [33]. A study on the fluid intake of Brazilian teenagers found that soft drinks are the second most consumed beverage, accounting for $10 \%$ of teenagers' daily energy intake [34]. The relationship between the frequency of sugary drinks and caries has been clearly demonstrated in preschool children and adults [35], with limited data on adolescents. Here, we present the impact of sugary drink consumption on caries incidence based on the most recent data sample of Chinese teenagers. We confirm a statistically significant relationship between elevated sugary drink consumption and dental caries experience.

In this study, we also found that eating vegetables twice a day or more can reduce the risk of caries. This result is similar to that of previous reports. A study by Chin-En Yen et al. found that a high vegetable intake effectively reduced dental caries incidence among 182 children aged 3-6 years [36]. In addition, a study of female university students in Saudi Arabia showed that eating vegetables four times per week or more effectively reduced caries risk (odds ratios $=0.73$ ) [37]. Another study conducted by Japanese scholars including 600 cases of 70-year-old subjects found that vegetable intake was negatively correlated with dental caries incidence [38]. Based on these findings, we speculate that vegetable intake is significantly associated with fewer dental caries, regardless of age.

Our data showed that approximately $20 \%$ of participants did not eat breakfast every day. There were significantly impaired eating habits that increased with age, including irregular breakfasts among 12- to 14- year-olds. Interestingly, we found evidence of "recovery" from these patterns and improved eating habits among 15-year-olds, as shown in supplementary Figure 1. This data trend agrees with that previously reported by Bruno et al. [15]. In addition, we found a statistically significant association between irregular breakfast patterns and caries development, with a 1.124 times increased caries risk. Skipping meals may reduce exposure to cariogenic foods, such as breakfast cereals, but irregular meal patterns, like light meals and high-sugar snacks throughout the day [15, 39], may explain the enhanced caries activity. In this study, caries risk caused by irregular breakfasts was lower than that reported in previous studies of Swedish female teenagers [15] and Polish preschool children [16]. This may be due to differences in the age and gender of the study populations or due to the differences in the diets of Eastern and Western people. Westerners generally consume more sugar in their daily diets than Easterners [40], and thus, Westerners may have a higher dental caries risk than Easterners.

However, our study has some limitations. We used a food frequency questionnaire to determine food intake frequency, but subjects may not be able to recall exactly what they have eaten over time. A food diary may be a solution to this problem, though it relies on the subject's compliance. Recently, pre-post meal digital photography proved to be a valid, accurate, and applicable method to assess food intake [41]. In addition, this study was a cross-sectional investigation, and the time course was not considered. Other studies have reported that previous dental caries experience at baseline increases the likelihood of future caries [42]. Thus, a longitudinal study with full consideration of baseline variables is required to 
confirm the effect of irregular breakfasts, sugary drinks, and vegetable intake on the occurrence of dental caries in the Chinese population.

\section{Conclusion}

The present study demonstrated a significant association between dietary habits and caries occurrence among adolescents aged 12-15 years in Shanghai. The caries prevalence among 12- to 15-year-old school students in Shanghai was lower than levels reported nationally and in some other developing or developed countries. Poor eating habits (higher sugary drink intake, lower vegetable intake, and skipping breakfast) in the developmental age may be a significant caries coefficient in adolescents.

\section{Declarations}

\section{Ethics approval and consent to participate}

This study was approved by the Independent Ethics Committee of Shanghai Municipal Center for Disease Control and Prevention in accordance with the Declaration of Helsinki. Participation in the study was voluntary and anonymous, and the collected data was treated confidentially, and a written informed consent form was sent to the parents of each child to explain the content of the survey. Each parent returned a signed informed consent form.

\section{Consent for publication}

Data collection was anonymous. No images or other personal details of participants are presented here. The patients were informed about the details collected from them and consent was taken for the use of their details in a scientific publication.

\section{Availability of data and materials}

The datasets used and/or analyzed during the current study is not publicly available due to the vulnerable study population囚but data available from the corresponding author on reasonable request.

\section{Competing interests}

The authors declare that they have no competing interests as defined by BMC, or other interests that might be perceived to influence the results and/or discussion reported in this paper.

\section{Funding}

This research was financially supported by the projects of Shanghai Municipal Commission of Health and Family Planning Foundation (201640149).

\section{Authors' contributions}


$X L Z, Y Z$ and HXX conceived, conceptualized and designed the study. HXX performed data analysis and wrote this manuscript. $\mathrm{HZ}$ established the database, and guided the data analysis. CYL, XGF, YWJ, JY, HNW, DXD, DLY, LJS conducted the survey and data collection. All the authors read, revised and approved the final manuscript.

\section{Acknowledgements}

The authors thank the adolescents and their parents for participation in the study.

\section{References}

1. Selwitz RH, Ismail Al, Pitts NB: Dental caries. Lancet (London, England) 2007, 369(9555):51-59.

2. Zeng L, Zeng Y, Zhou Y, Wen J, Wan L, Ou X, Zhou X: Diet and lifestyle habits associated with caries in deciduous teeth among 3-to 5-year-old preschool children in Jiangxi province, China. BMC oral health 2018, 18(1):224.

3. Smits KPJ, Listl S, Jevdjevic M: Vegetarian diet and its possible influence on dental health: A systematic literature review. Community dentistry and oral epidemiology 2020, 48(1):7-13.

4. Paglia L, Scaglioni S, Torchia V, De Cosmi V, Moretti M, Marzo G, Giuca MR: Familial and dietary risk factors in Early Childhood Caries. European journal of paediatric dentistry 2016, 17(2):93-99.

5. Morikava FS, Fraiz FC, Gil GS, de Abreu M, Ferreira FM: Healthy and cariogenic foods consumption and dental caries: A preschool-based cross-sectional study. Oral Dis 2018, 24(7):1310-1317.

6. Hujoel PP, Lingström P: Nutrition, dental caries and periodontal disease: a narrative review. Journal of clinical periodontology 2017, 44 Suppl 18:S79-s84.

7. Sharma B, Indushekar KR, Saraf BG, Sardana D, Sheoran N, Mavi S: Are dental caries and overweight/obesity interrelated? A cross-sectional study in rural and urban preschool children. Journal of the Indian Society of Pedodontics and Preventive Dentistry 2019, 37(3):224-231.

8. Moynihan PJ, Kelly SA: Effect on caries of restricting sugars intake: systematic review to inform WHO guidelines. J Dent Res 2014, 93(1):8-18.

9. Barrington G, Khan S, Kent K, Brennan DS, Crocombe LA, Bettiol S: Obesity, dietary sugar and dental caries in Australian adults. International dental journal 2019, 69(5):383-391.

10. Abbass MMS, AbuBakr N, Radwan IA, Rady D, El Moshy S, Ramadan M, Ahmed A, Al Jawaldeh A: The potential impact of age, gender, body mass index, socioeconomic status and dietary habits on the prevalence of dental caries among Egyptian adults: a cross-sectional study. F1000Research 2019, 8:243.

11. Park S, Lin M, Onufrak S, Li R: Association of Sugar-Sweetened Beverage Intake during Infancy with Dental Caries in 6-year-olds. Clinical nutrition research 2015, 4(1):9-17.

12. Newbrun E: Sugar and dental caries: a review of human studies. Science (New York, NY) 1982, 217(4558):418-423. 
13. Monteagudo C, Téllez F, Heras-González L, Ibañez-Peinado D, Mariscal-Arcas M, Olea-Serrano F: SCHOOL DIETARY HABITS AND INCIDENCE OF DENTAL CARIES. Nutricion hospitalaria 2015, 32(1):383-388.

14. Elamin A, Garemo M, Gardner A: Dental caries and their association with socioeconomic characteristics, oral hygiene practices and eating habits among preschool children in Abu Dhabi, United Arab Emirates - the NOPLAS project. BMC oral health 2018, 18(1):104.

15. Bruno-Ambrosius K, Swanholm G, Twetman S: Eating habits, smoking and toothbrushing in relation to dental caries: a 3-year study in Swedish female teenagers. International journal of paediatric dentistry 2005, 15(3):190-196.

16. Krzywiec E, Zalewska M, Wójcicka A, Jabłoński R, Olejnik BJ, Grabowska SZ, Jamiołkowski J, Czerech E, Łuszcz A, Stepek A et al: [Selected eating habits and caries occurrence in adolescents]. Przeglad epidemiologiczny 2012, 66(4):713-721.

17. Smith MA, Wells MH, Scarbecz M, Vinall CV, Woods MA: Parents' Preferences and Perceptions of Their Children's Consumption of Sugar and Non-nutritive Sugar Substitutes. Pediatric dentistry 2019, 41(2):119-128.

18. Editors PM: Observational studies: getting clear about transparency. PLoS Med 2014, 11(8):e1001711.

19. Zhang H, Zeng $X$, Jiang $Y, X u$ W, Wang X, Li C, Zhang Y, Liu Y, Wang Y: The disparity in caries and sealants between migrant and native children in Shanghai: A cross-sectional study. International journal of dental hygiene 2020, 18(1):84-91.

20. Aimée NR, van Wijk AJ, Maltz M, Varjão MM, Mestrinho HD, Carvalho JC: Dental caries, fluorosis, oral health determinants, and quality of life in adolescents. Clinical oral investigations 2017, 21(5):18111820.

21. Feng X: Oral Health Status of Chinese Residents--The Fourth National Oral Health Epidemiological Survey. In: 2018 Chinese Academy of Stomatology The 18th Annual Conference of Dental Preventive Medicine: 2018; Xi'an, Shaanxi Province, China; 2018: 2.

22. Hu J, Jiang W, Lin X, Zhu H, Zhou N, Chen Y, Wu W, Zhang D, Chen H: Dental Caries Status and Caries Risk Factors in Students Ages 12-14 Years in Zhejiang, China. Med Sci Monit 2018, 24:3670-3678.

23. Yin W, Yang YM, Chen H, Li X, Wang Z, Cheng L, Yin QD, Fang HZ, Fei W, Mi FL et al: Oral health status in Sichuan Province: findings from the oral health survey of Sichuan, 2015-2016. International journal of oral science 2017, 9(1):10-15.

24. Goel R, Vedi A, Veeresha KL, Sogi GM, Gambhir RS: Oral hygiene practices and dental caries prevalence among 12 \& 15 years school children in Ambala, Haryana -A cross-sectional study. J Clin Exp Dent 2015, 7(3):e374-379.

25. Obregón-Rodríguez N, Fernández-Riveiro P, Piñeiro-Lamas M, Smyth-Chamosa E, Montes-Martínez A, Suárez-Cunqueiro MM: Prevalence and caries-related risk factors in schoolchildren of 12- and 15year-old: a cross-sectional study. BMC oral health 2019, 19(1):120. 
26. Milciuviene S, Bendoraitiene E, Andruskeviciene V, Narbutaite J, Sakalauskiene J, Vasiliauskiene I, Slabsinskiene E: Dental caries prevalence among 12-15-year-olds in Lithuania between 1983 and 2005. Medicina (Kaunas, Lithuania) 2009, 45(1):68-76.

27. Ferraro $M$, Vieira AR: Explaining gender differences in caries: a multifactorial approach to a multifactorial disease. International journal of dentistry 2010, 2010:649643.

28. Polk DE, Geng M, Levy S, Koerber A, Flay BR: Frequency of daily tooth brushing: predictors of change in 9- to 11-year old US children. Community dental health 2014, 31(3):136-140.

29. Bernabe E, Newton JT, Uutela A, Aromaa A, Suominen AL: Sense of coherence and four-year caries incidence in Finnish adults. Caries research 2012, 46(6):523-529.

30. Rothen M, Cunha-Cruz J, Zhou L, Mancl L, Jones JS, Berg J, Northwest Pn: Oral hygiene behaviors and caries experience in Northwest PRECEDENT patients. Community dentistry and oral epidemiology 2014, 42(6):526-535.

31. Ekuni D, Tomofuji T, Mizutani S, Furuta M, Irie K, Azuma T, Kojima A, Iwasaki Y, Morita M: Dental caries is correlated with knowledge of comprehensive food education in Japanese university students. Asia Pacific journal of clinical nutrition 2013, 22(2):312-318.

32. Attin $T$, Hornecker $E$ : Tooth brushing and oral health: how frequently and when should tooth brushing be performed? Oral health \& preventive dentistry 2005, 3(3):135-140.

33. Lee S, Park YJ, Kim M, Lee HK, Han K, Kang CS, Kang MW: Prevalence of Ambler class A and D betalactamases among clinical isolates of Pseudomonas aeruginosa in Korea. The Journal of antimicrobial chemotherapy 2005, 56(1):122-127.

34. Feferbaum R, de Abreu LC, Leone C: Fluid intake patterns: an epidemiological study among children and adolescents in Brazil. BMC public health 2012, 12:1005.

35. Armfield JM, Spencer AJ, Roberts-Thomson KF, Plastow K: Water fluoridation and the association of sugar-sweetened beverage consumption and dental caries in Australian children. American journal of public health 2013, 103(3):494-500.

36. Yen CE, Huang YC, Hu SW: Relationship between dietary intake and dental caries in preschool children. International journal for vitamin and nutrition research Internationale Zeitschrift fur Vitaminund Ernahrungsforschung Journal international de vitaminologie et de nutrition 2010, 80(3):205-215.

37. Musaiger AO, Al-Mannai M, Abduljawad E: Association of oral hygiene habits and food intake with the risk of dental caries among undergraduate university women in Saudi Arabia. International journal of adolescent medicine and health 2014, 26(4):585-589.

38. Yoshihara A, Watanabe R, Hanada N, Miyazaki H: A longitudinal study of the relationship between diet intake and dental caries and periodontal disease in elderly Japanese subjects. Gerodontology 2009, 26(2):130-136.

39. Dwyer JT, Evans M, Stone EJ, Feldman HA, Lytle L, Hoelscher D, Johnson C, Zive M, Yang M: Adolescents' eating patterns influence their nutrient intakes. Journal of the American Dietetic Association 2001, 101(7):798-802. 
40. Ha K, Chung S, Joung H, Song Y: Dietary sugar intake and dietary behaviors in Korea: a pooled study of 2,599 children and adolescents aged 9-14 years. Nutrition research and practice 2016, 10(5):537545.

41. Winzer E, Luger M, Schindler K: Using digital photography in a clinical setting: a valid, accurate, and applicable method to assess food intake. European journal of clinical nutrition 2018, 72(6):879-887.

42. Evans RW, Clark P, Jia N: The Caries Management System: are preventive effects sustained postclinical trial? Community dentistry and oral epidemiology 2016, 44(2):188-197.

Figures

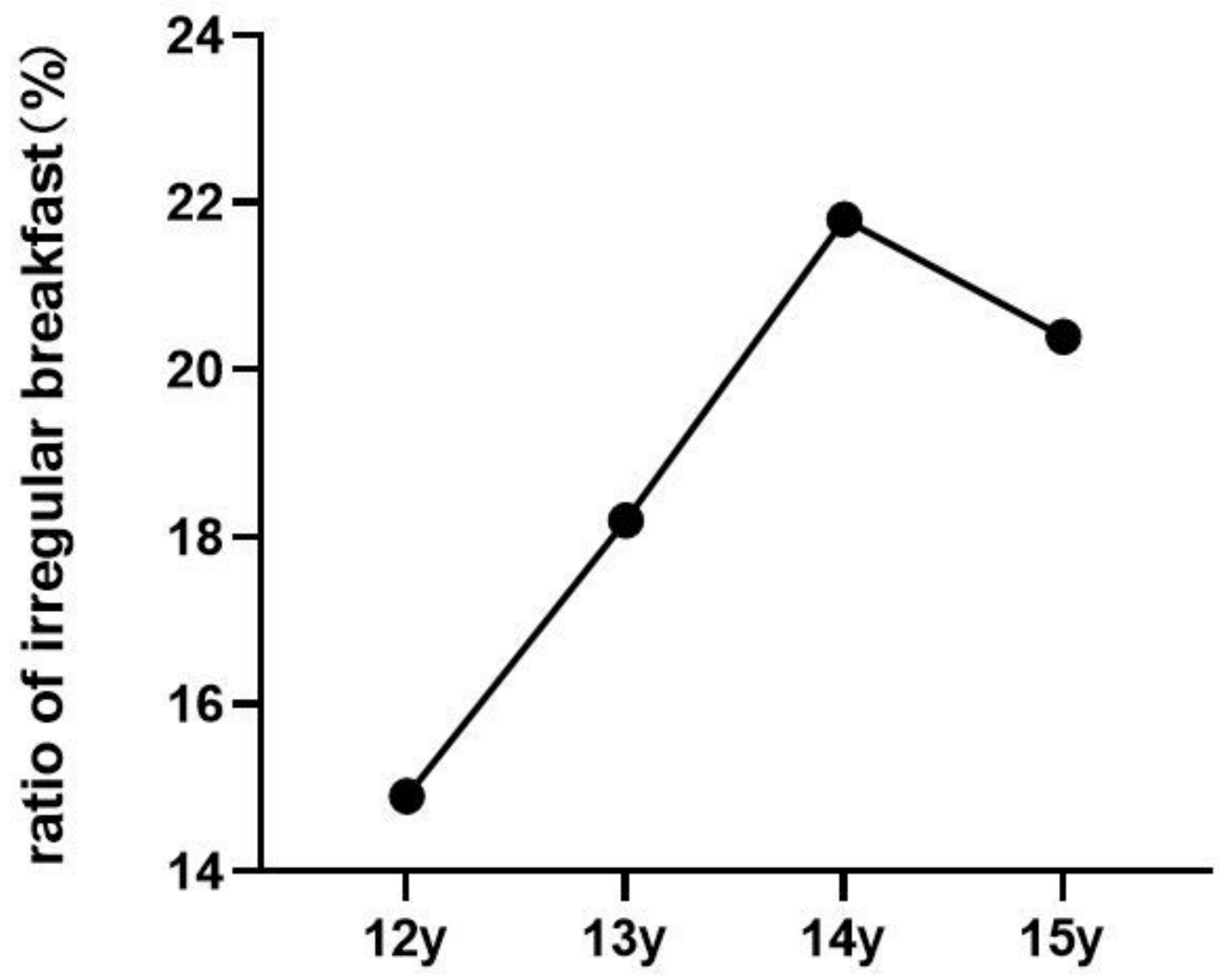

Figure 1

Irregular breakfast with age 